University of New Hampshire

University of New Hampshire Scholars' Repository

\title{
$5-2015$
}

\section{A Bayesian marine debris detector using existing hydrographic data products}

\author{
Giuseppe Masetti \\ University of New Hampshire, Durham, giuseppe.masetti@unh.edu \\ Brian R. Calder \\ University of New Hampshire, Durham, brian.calder@unh.edu
}

Follow this and additional works at: https://scholars.unh.edu/ccom

\section{Recommended Citation}

G. Masetti and Calder, Brian R., "A Bayesian marine debris detector using existing hydrographic data products", IEEE Oceans. IEEE, Genoa, Italy, 2015.

This Conference Proceeding is brought to you for free and open access by the Center for Coastal and Ocean Mapping at University of New Hampshire Scholars' Repository. It has been accepted for inclusion in Center for Coastal and Ocean Mapping by an authorized administrator of University of New Hampshire Scholars' Repository. For more information, please contact Scholarly.Communication@unh.edu. 


\title{
A Bayesian marine debris detector using existing hydrographic data products
}

\author{
Giuseppe Masetti ${ }^{1, *}$ and Brian Calder ${ }^{2, *}$ \\ ${ }^{1}$ Tel: +1-(603)-8623452, email: gmasetti@ccom.unh.edu; ${ }^{2}$ email: brc@ccom.unh.edu
}

${ }^{*}$ Center for Coastal and Ocean Mapping \& Joint Hydrographic Center, University of New Hampshire, USA

Natural disasters can represent a massive source of marine debris deposition along the coastline due to strong winds, heavy rainfall, and storm surge. Such an extensive amount of debris of different size, shape, and materials could threaten navigation, natural resources, and/or human safety. After a disaster (e.g., hurricane, typhoon, tsunami, etc.), effectively and quickly processing large amounts of hydrographic data, collected using commercial systems for detection and classification of marine debris, would be highly advantageous to the necessary remediation operations. Such a task involves some degree of modeling and approximation to make the analysis computationally attractive and sufficiently effective in practice (i.e., an approximate solution with a wellstructured model is preferred to an exact solution with a suboptimal model).

For this reason, a target model was built postulating a simplified description of the object properties, and a detector was specifically outlined for marine debris, detecting discrete objects which differ (e.g., protrude) from the surrounding seafloor, being close or connected to the bottom. The scope of the detector was also constrained to analyze products commonly available in existing post processing software (mainly, bathymetric digital terrain models (DTM) and backscatter mosaics with several associated data sets, such as statistics derived from the core data, or during construction) so that the technique may be quickly inserted into existing workflows, which eases resource management in a response situation.

For the backscatter mosaic, as in many existing algorithms, target detection is based on the observation that denser material (often anthropogenic) makes debris returns much stronger than the surrounding background. However, the often used approach to object detection through simple thresholding (e.g., based on the premise that on a mosaic the object return is brighter than the background) was modified since it tends to fail when the background is textured (i.e., detectors are not aware of image correlation). For the bathymetric DTM, a few spatial indices were used as proxies for any discontinuity and, thus, a possible target. For both backscatter and bathymetry, the adoption of classical estimation techniques usually generates point estimate or a confidence interval, which becomes important when fusion of target information coming from different products is attempted. In order to provide appropriate distributions for exploitation, Bayesian methods were adopted since they permit use of multiple-source asymmetric and discontinuous posterior distributions that may be carried into further analysis. A hierarchical scheme is proposed where a series of modeling tasks are implemented through a probabilistic model, casting the debris detection problem as one of estimating properties of the posterior 
distribution describing the probability of objects occurring given the observed data products.

The proposed model hypothesizes that the observed products are formed through observations of naturally smooth processes, modified to incorporate the presence of potential objects. Pragmatic advantages of the proposed Bayesian framework are the opportunity to include prior knowledge as well as developing an extendible robust scheme for estimating the probability of an object occurring at any point in the area of interest, making detection simpler. For instance, additional background information can be added as it becomes available. By inspection on existing nautical charts, the backscatter return far from marine debris can be approximated using a texture model or, in case the area has a geological context of large scatterers, a default model providing less informative priors.

A Markov chain Monte Carlo (MCMC) system is used to estimate probability of object presence on a per pixel/node basis. The data probability under the hypothesis of having marine debris is produced by the detector from different types of products, related both to bottom detection (depth) and to the intensity time series usually collected over it (backscatter). Together with the updated posterior probabilities, the Bayesian estimator maintains a binary detection layer. Such a layer provides a means to partially vectorize the analysis, i.e. applying prior constraints through computational geometry, and can also be used as the basis for higher-order (e.g., object-based) constraints or detection modeling.

Finally the paper provides some examples of debris detections based on the analysis of real data collected after the Super Storm Sandy event, and the detector outcomes are shown in order to evaluate the effectiveness of the proposed technique. Since the framework provides sufficient flexibility to integrate additional source of information, we are currently working on extending the detector with a set of ad hoc hydrographic products with the intent to improve the detection performance. 